\title{
Web application for self-diagnosis and drug recommen- dation based on user symptoms
}

\author{
Iuliana Marin ${ }^{1}$, Nicolae Goga ${ }^{2 *}$, Razvan-Constantin Stanciu ${ }^{3}$ \\ 1, 2, 3 Department of Engineering in Foreign Languages, University Politehnica of Bucharest, Bucharest, Romania
}

\author{
Keywords \\ Online self-diagnosis \\ Medication recommender system \\ Symptoms \\ Diseases \\ Big data \\ Data mining
}

Received: 5 February 2019

Accepted: 6 March 2019

Published: 20 April 2019

\begin{abstract}
Throughout the evolution of the Internet and social networks, forums and online platforms have a vital role in sharing information, along with the creation and engagement of virtual communities. Such websites represent great resources, and they are the first step in the adoption of e-health services. When the persons are ill, many of them use search engines for self-diagnosis and gather possible treatment ideas before asking for a doctor's opinion. This takes a lot of time because the information is scattered across various forums and websites. In this paper is presented an application that aims to provide an online self-diagnosis and drug recommendation tool based on natural language processing of the symptoms described by the user. Over 2,200 medicines are stored in the database, each having a set of keywords according to their usage. Thus, the platform automates the search process, and provides the user with the most relevant information, eliminating the need for manual data interpretation. The results are ranked according to the confidence score obtained after the execution of the fuzzy search algorithm. The platform does not provide medical advice. Thus it is intended for informational purposes only. The developed platform is not a substitute for professional medical advice, diagnosis or treatment. Another feature of the platform is that it enables users to find hospitals and clinics around them, so that they can receive professional healthcare service. The field of medicine is a sensitive topic due to the fact that one mistake could lead to the loss of countless human lives. It was a long and difficult journey to reach the current state of medicine, but technology was and will remain essential to the evolution of the healthcare system. The proposed solution of this paper is customized for Romania but it can be adapted for other countries by replacing the drug database.
\end{abstract}

(c) 2019 The Author(s). Published by TAF Publishing.

\section{INTRODUCTION}

Throughout the evolution of the internet and social networks, forums and online platforms had a vital role in sharing information and creating engaging communities. These websites represent great resources, and they are the first step in the adoption of e-health services. People use forums so that they can freely ask questions about their health conditions and talk about possible treatments with other users having similar experiences $[1,2]$. Modern technology allows us to collect the information found on these platforms. This valuable information can be then used to create smart applications such as self-diagnosis systems, medicine recommender systems, e-Doctor websites, cloud-assisted drug recommendation services, and many others $[3,4]$.
Building a self-diagnosis system is a complicated task, and one should know that there are various challenges to overcome. One such problem is the accuracy of the system. Accurate diagnosis of a disease is a complicated process that has to consider many factors. There were many attempts to build such a system. However, the majority of them failed to be deployed for practical use.

There are many approaches related to the idea of a selfdiagnosis system. Some methods have the goal to altogether remove the intervention of the medical expert from the decision-making process, while others are considered support systems and only speed up the process by offering a valuable interpretation of the data for the human expert to analyze.

\footnotetext{
${ }^{*}$ Corresponding author: Nicolae Goga

†email: n.goga@rug.nl
} 
In this paper, we propose to define the model of a web application for self-diagnosis and drug recommendation, which is based mainly on healthcare keywords. This comes as an answer to the lack of medicine recommendation systems in online self-diagnosis applications. In the next section, we present the literature review. In section III, we describe the architecture of the application and the main algorithm. Section IV contains the final results, illustrating the functionalities of the system. In the end, we conclude and discuss further ways to improve our solution.

\section{RELATED WORK}

During recent years, the healthcare domain went through a great number of transformations thanks to advances in technology. Today, people are more interested in their health condition, so they spend more time online, searching for health-related subjects and information. This kind of active interest from patients led to the enhancement of the doctor-patient relationship and improved service engagement. The influence of technology is obvious in this case, and it helps in co-creating value in the medical domain at the micro level [5].

Medical records contain large sets of data such as patient information, medical history, drug prescriptions, treatment protocols and outcomes [6]. The amount of unstructured electronic health data is hard to manage and analyze using traditional ways. Today, big data is an emerging trend in technology that is used to describe a large volume of structured and unstructured data sets in order to extract valuable information. The information mined can be then used in various machine learning applications or analytics projects. The healthcare industry is behind other fields when it comes to the use of big data. This is not a surprise given the nature of the domain and the challenges faced by the healthcare providers. There is no simple way to share data among the different providers without causing security and privacy concerns. Big data is still in its early days and there is potential to develop applications that can bring value to the medical industry [7].

In order to efficiently process large data sets and provide a real-time response, cloud-computing is a great choice to enhance the computing performance of the system. For example, Apache Hadoop and Apache Spark are two excellent cloud platforms designed for data mining with the possibility of iterative computation. Apache Spark supports parallel programming models such as Resilient Distributed Datasets (RDDs) and Directed Acyclic Graphs (DAGs) so that iterations on the same dataset can be performed directly from memory where the data caches are saved.
The majority of self-diagnosis systems are made of two core modules. The first one is the disease-symptom clustering analysis module, in which the symptoms are classified based on given reports. Disease diagnosis and drug recommendation service is the second module of the system [8]. A knowledge base is used to save knowledge from human experts by structuring the information in facts and rules. The reasoning engine is responsible for the decisionmaking process so that users can receive professional opinions through a virtual knowledge base. This type of system is also called an expert system [9]. An expert system can use Fuzzy logic or Boolean logic. In a fuzzy expert system, the truth values of the variables can be any real number between 0 and 1.

The way the application works is easy to understand. The user has to indicate the area of interest (problem area) on a virtual human body mannequin. Then, based on their selection, users have to select symptoms from a list. In the end, they are asked a few questions and according to their answers, the expert system diagnosis diseases based on its knowledge base. Finally, the algorithm ranks the results and display them in a fuzzy form $[10,11,12]$. Another approach to achieve an automatic medical diagnosis system is e-Doctor. This kind of web application is based on Support Vector Machines (SVM). SVMs are models trained through supervised learning that can analyze and recognize patterns in data and make decisions, based on their knowledge base. This type of system is used in clinics, where the doctors can insert data about patients or their examinations and the edoctor will make predictions about the current or future health status of the patient. The SVM learning strategy is a powerful technique that can be used in applications such as image segmentation and classification, image fusion and object recognition [13].

Recommender systems first started to receive attention from the IT domain in the mid-1990s. Following extended research, experts immediately realized the utility of such a system $[14,15]$. Nowadays, e-commerce is a growing business and recommender systems have become popular when it comes to online shopping [16]. More people tend to buy medicine online for convenience reasons. However, purchasing medication without professional guidance can become an issue. Although online pharmacies offer detailed information about the drugs and even user ratings, there are two important problems to be considered when buying medication online:

- Validity-There is a chance of purchasing the wrong or a noneffective drug because of the misleading or exaggerated information on the platform. 
- Reliability-Without professional instruction, people do not pay attention to the interaction of multiple medicines which can cause serious issues for the patients.

There are various types of drug recommendation systems. For example, in a cloud-assisted medication recommender system, users are presented with top-N related drugs based on their described symptoms. The algorithm also takes into consideration factors such as when the cold started and the type of the drug [17]. Data mining techniques can also be applied to the recommendation system to decrease medical errors. Other modules such as the database module, model evaluation module, recommendation model module, and data preparation module can be found in a recommender system.

Other types of recommender systems use a list of risk factors that the doctor must fulfill regarding the patient's health. Then, the system will compute the risk level of the patient and the doctor can select a possible bacterium. The recommended drugs are listed in order of priority [18].

An example of an ontology-based drug recommendation system is GalenOWL. The purpose of GalenOWL is to bind semantic web and medical terminologies so that it can respond to queries related to drug recommendations. The user sends requests to the system in order to find drug indications and contraindications according to the patient data. All the information about the patient is used to build the knowledge base then, the rule-based reasoning is done. Medical ontologies are used by the reasoning engine in order to return the list of drug recommendations.

Data mining is a great enhancement to the development of a recommender system. It has the potential to highlight hidden relationships and patterns between medical data sets. These patterns can be used to improve medical diagnosis and drug recommendation services by providing a better understanding of the interaction between drugs and diseases [19].

In the world of computer science, Artificial Intelligence (AI) refers to the development of computer systems that are able to demonstrate machine intelligence. These systems are able to complete tasks that normally require human intellectual and decision-making [20]. The invention of robots is considered the starting point in the development of artificial intelligence. The word 'robot' is derived from Czech ('robota'), which means biosynthetic machines used as forced labor. AI has a huge range of applications in the healthcare industry. From computer-assisted medical diagnosis and robotics to health recommender systems, all of these can be powered by artificial intelligence technology [21].
AI in Medicine (also known as AIM) started as a US research community. The goal was to innovate the world of medicine and overcome the limitations of technology. The healthcare domain remains an attractive field for researches all around the globe because it can provide them with a diverse set of real-world problems that they can work on [22]. In medicine, the field of $\mathrm{AI}$ is divided into two main categories - virtual and physical. The virtual part contains Machine Learning (ML) and algorithms. There are three types of ML algorithms: a) unsupervised learning; b) supervised learning; and c) reinforcement learning. The physical component includes robots and other medical devices [22].

AI together with the latest data mining tools can become key elements in the prediction of medical outcomes. By using machine learning algorithms, one can develop powerful applications containing accurate classifiers. For classification and prediction modeling queries, it is important to provide accurate training data when dealing with medical records [23].

There is no secret that big data will revolutionize medicine. The majority of computer-based systems in healthcare are expert systems. They contain a knowledge base with different rule sets encoded. The name comes from the fact that those systems know the general principles of medicine and their mission is to apply them to new patients [24].

$\mathrm{AI}$ is an emerging technology that currently lacks the trust of humans. Patients want to feel that investigations are conducted by doctors and not by machines. The role of artificial intelligence is to take advantage of the computing power and leave the management of the patient in the hands of the doctors [25]. There is a debate going on, whether AI systems are actually better than human professionals, but everyone can agree on the fact that mixing machine learning software with the knowledge of the human experts will outperform what each of them can achieve on their own [26]. Although one should not neglect the benefits of deep learning in healthcare, there are still several challenges left to be solved. Data volume is one such challenge.

The amount of medical data required to train the model efficiently is much greater than in any other field. Furthermore, data quality is a serious issue in medicine. Unlike other domains, it is difficult to find well-structured data to use for model training [27].

The integration of new technologies and ideas in the medical industry also introduces tough challenges that need to be addressed. Nowadays, cloud computing is gaining massive popularity. This approach brings important benefits such as flexibility, scalability and it is cost effective. Implementing cloud-based applications in the healthcare domain 
is not an easy task and has led to security and privacy concerns because the patient data is at stake. Such data contain sensitive records and unauthorized access must not be allowed at any cost. In order to avoid a security breach, network administrators have to continuously analyze and patch the security vulnerabilities of the wireless networks before they become a threat [28]. They also have to look out for the possible loss of data in case of a disaster or an unexpected event and therefore implement automatic backup features. System unavailability is another cloud computing risk which has to be taken into consideration. Not being able to use health services in emergenc could have devastating effects on the population.

There are many types of challenges when it comes to implementing e-health (electronic health) services besides the technological ones. One way to classify those challenges is the following:

- Financial challenges-Funding is a difficult challenge because there are high costs in order to implement, monitor and provide technical support for such a system.

- Socio-cultural challenges-Refers to the negative attitude towards the concept of e-health due to factors such as peers influence, fear of losing the interactions with the medical staff, poverty, religious beliefs and age factors.

- Human challenges-Describes the shortage of health workers with the required IT skills and knowledge to understand and use e-health systems.

- Legal challenges-Presents law issues which include the lack of policies and legal pressure on healthcare providers when it comes to information sharing.

Overcoming the existing technological and human barriers is necessary for the development of the medical field. Scientists, engineers, and medical experts will always try to push the boundaries of medicine and increase the lifespan of human beings.

Today, online health platforms are increasing in popularity because there is no need for the doctor to be in the same area as the patient. The issue related to resources can now be solved as more doctors and patients are using e-health services to eliminate the time and distance barriers.

\section{METHOD}

Patolo is the name of the solution proposed in this paper. The aim of the application is to provide an online selfdiagnosis and drug recommendation tool based on symptoms described by the user.

It is difficult to build a reliable, easy-to-use medical platform, in order for users with no prior medical knowledge to be able to quickly find if they suffer from a common disease and which medicine to use as a treatment. Patolo is based on keywords.

The application was designed to look and feel like a healthcare-related search engine, so that users describe their issues in a natural way. The only drawback of this approach is that, like any other search engine, the results will vary depending on the description.

In the following paragraphs, the features, architecture and algorithm behind Patolo will be presented and explained.

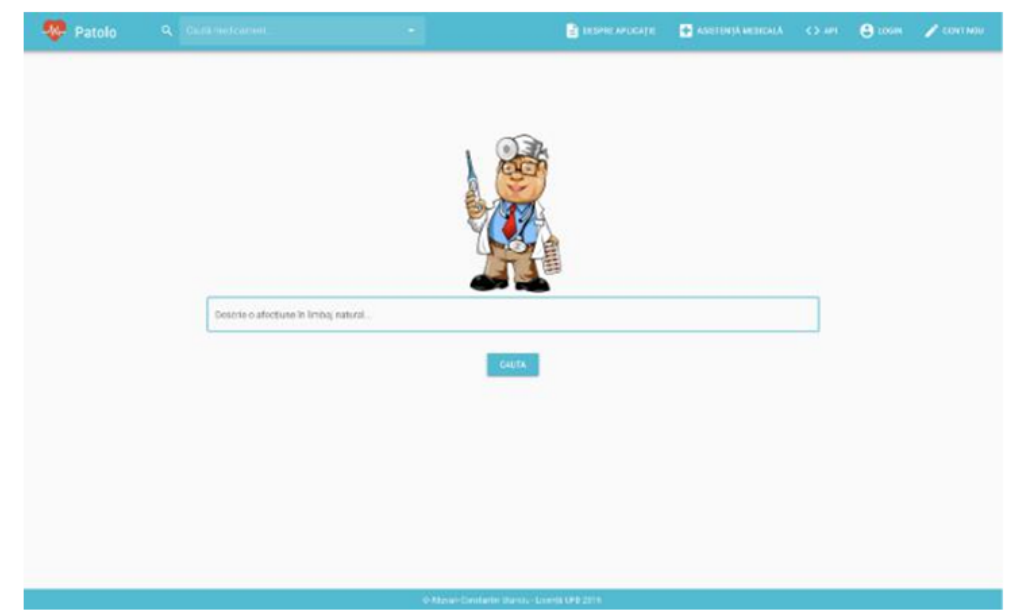

Fig. 1. Patolo-application homepage

\section{A. Features}

In addition to the self-diagnosis and drug recommendation tool, the mission of the application is to provide useful online healthcare features so that users can be well-informed.
- Self-diagnosis and medication recommendation based on user symptoms-this gives the user the freedom to describe his/her symptoms in natural language and receive a list of possible medical conditions and treatment suggestions.

- Search for a medicine-it is essential to be well-informed 
and this gives the users the option to find and read about a particular medicine.

- Read about a disease-this offers the user the ability to find out information about the diseases that are displayed at the end of the diagnosis procedure.

- Find medical assistance-this feature allows the users to see a map containing hospitals and clinics around them, so that they can get professional healthcare assistance.

- Account registration-registered users have additional benefits like the option to add medicines to favorite or even vote for a medicine.

- Medicines public API-this feature is written so that developers can use the medicines from the database in order to build other medical applications. This will save them a lot of time and resources from having to make their own database.

\section{B. Algorithm}

The algorithm behind the self-diagnosis and drug recommendation feature is divided in three stages. Initially, after the user describes the symptoms in natural language, the text will be translated from Romanian to English. This is achieved by using Google Translation Cloud service. The reason why the translation is required is explained in the next step of the algorithm.

In the second stage of the algorithm, the keywords extraction process is started. This means that from the previously translated description, medical keywords will be extracted (keywords that refer to symptoms, diseases or conditions). This is done by using a natural language processing algorithm specialized in healthcare. The best, free solution in this case is Amazon Comprehend Medical, part of the AWS. However, Romanian language is not supported and this is the reason why the translation is required.

In the last stage, once the keywords are obtained, a fuzzy search algorithm will attempt to match these keywords to the keywords of the medicines and also to the keywords of the diseases in the database. A threshold confidence score and a result limit will make sure that the performance of the fuzzy search algorithm is a good one. Results with a bad score are dropped immediately. The final results are sorted based on the confidence score. This way, users will know which are the relevant results to their query.

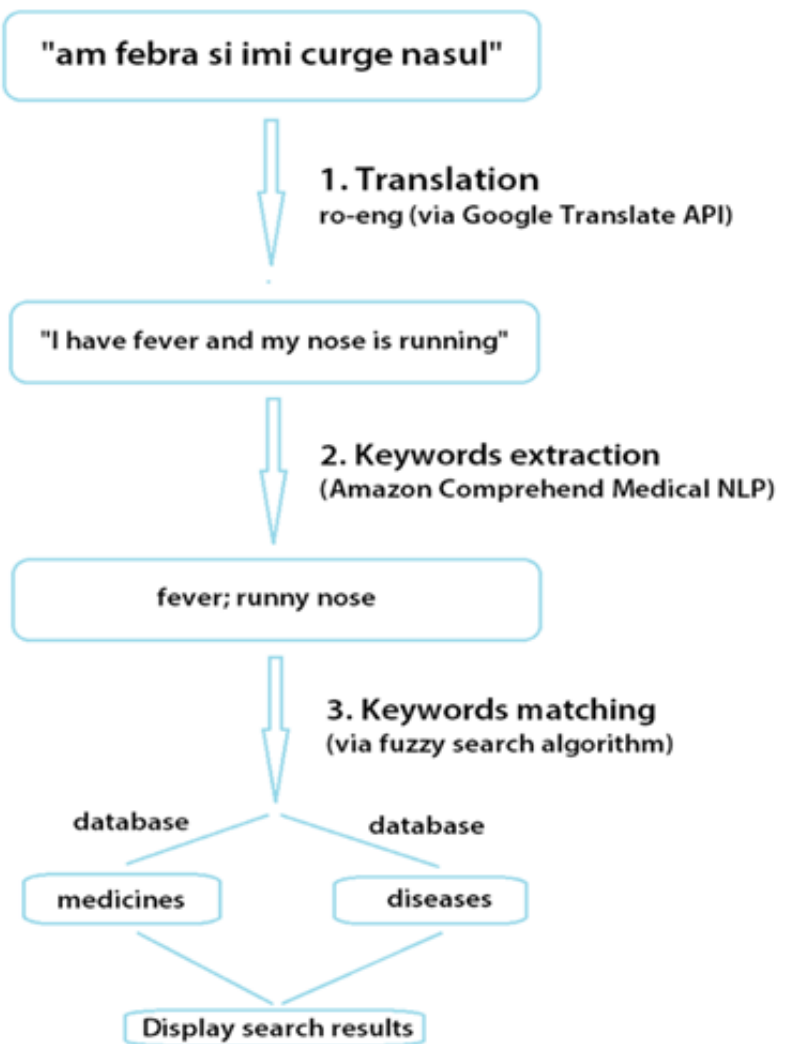

Fig. 2. Algorithm breakdown for main use case 
Rezultate cautare

Descriere afectiune: am febra si imi curge nasul

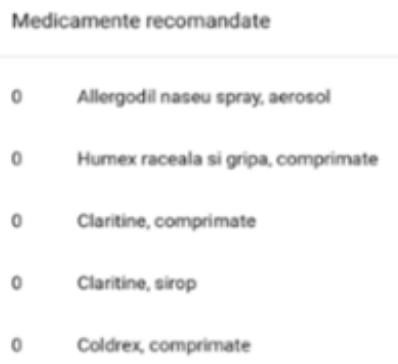

Fig. 3. Self-diagnosis query result

\section{Technologies and Architecture}

The application is running on a 3-tier web architecture. The technological stack used is called MEVN (mongoDB, express.js, Vue.js, Node.js).

On the front-end, we have a Vue.js client instance packed with Vuex for state management and Vuetify for material design components. The client is communicating with the back-end via REST APIs.When it comes to the back-end, Patolo is running Node.js with express.js as a web server. The last layer of the architecture, data storage is done via MongoDB, a great NoSQL solution to store structured or unstructured documents. The combination of those technologies results in a fast, elegant and modern looking application.

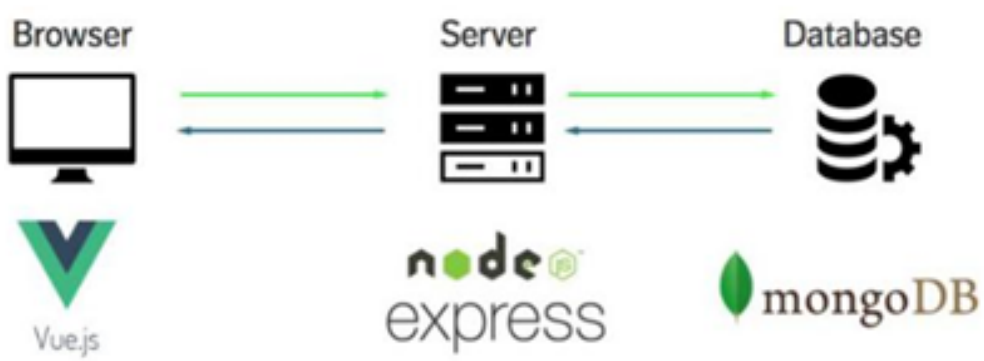

Fig. 4. Overview of the architecture

The figure above represents a simplified schematic of the main components in the architecture. In the following figures, the communication between the back-end and the front-end is described in details to understand the flow of the request. Picking the right technology stack and architecture was essential due to the nature of this application.
The architecture described above provides excellent performance in terms of computing speed and reliability. Having a large database, continuously expanding and using a fuzzy search algorithm, were indicators that an elegant data storage solution was required, thus the decision to use MongoDB.

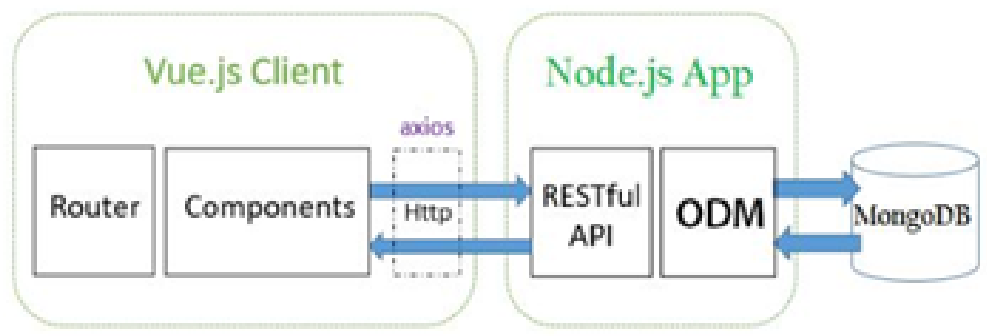

Fig. 5. Full-stack architecture overview 


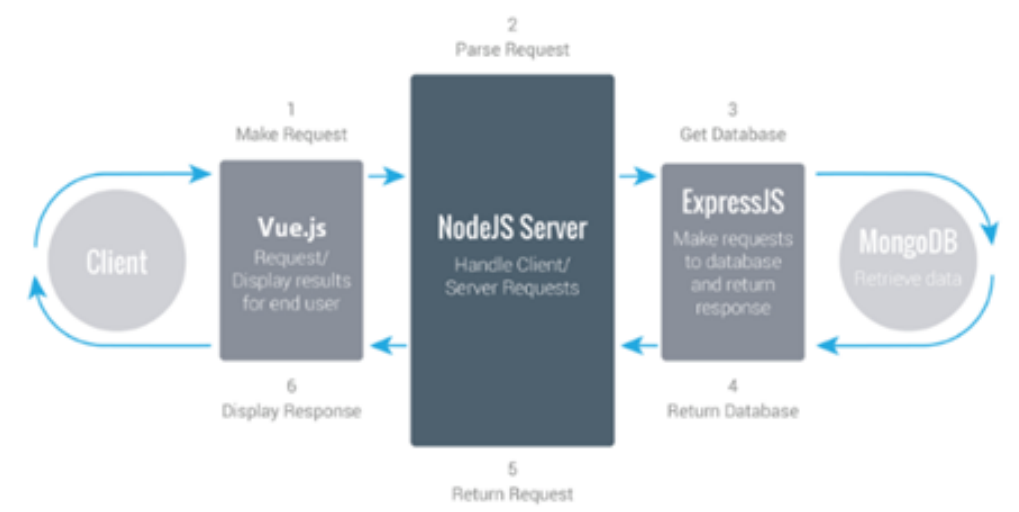

Fig. 6. Diagram describing flow of the requests

\section{RESULTS}

To compose a medical database out of unstructured raw documents is a tough task for any developer. After days of data mining and refinement, the application now has over 2,200 medicines in the database, each having keywords set according to their usage.

The main functionality behind Patolo is the self-diagnosis and drug recommendation engine. This allows people to use the website like a regular search engine and describe their issues in natural language in the same way that they would write on a forum.

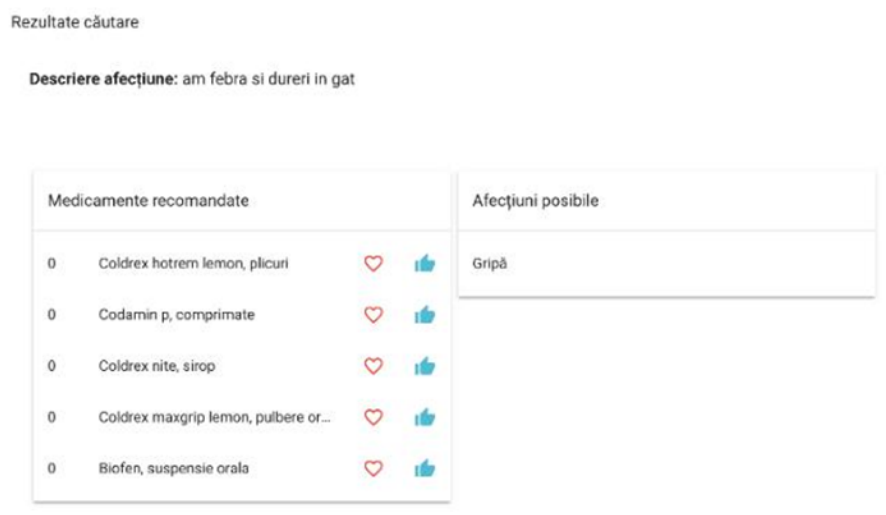

Fig. 7. Patolo-self-diagnosis query results

The results are ranked according to the confidence score obtained after the execution of the fuzzy search algorithm. User votes do not impact the ranking procedure.

By pressing the red, heart-shaped button, one can save that particular medicine to favorites. In addition, by clicking on the blue, thumbs up button, one can vote for that drug. A page with detailed information is displayed when either the name of a medicine or the name of a disease is clicked.

Users have the option to search for a medicine and read about it. The search form has autocomplete and suggestion features to help the user with the name of the drug.

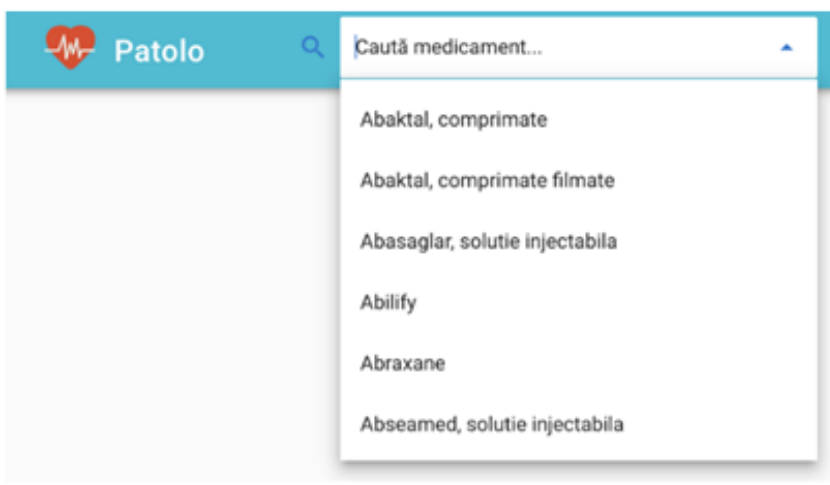

Fig. 8. Patolo-drug search form

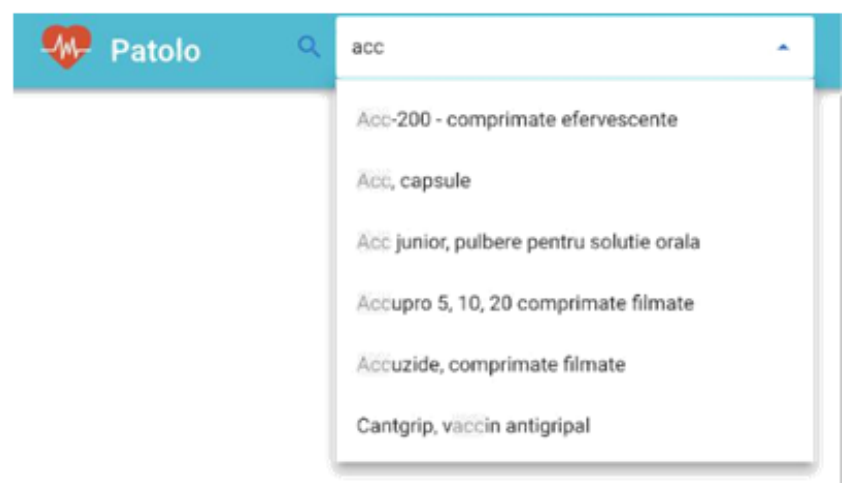

Fig. 9. Patolo-drug search autocomplete feature

Patolo does not provide medical advice. It is intended for informational purposes only. It is not a substitute for professional medical advice, diagnosis or treatment. This feature enables users to quickly find hospitals and clinics around them, so that they can receive professional healthcare service.

Registered users can access their profile page and find information regarding their account. Registration date and saved medicines are located there. They can click on the name of a drug to read about it. 


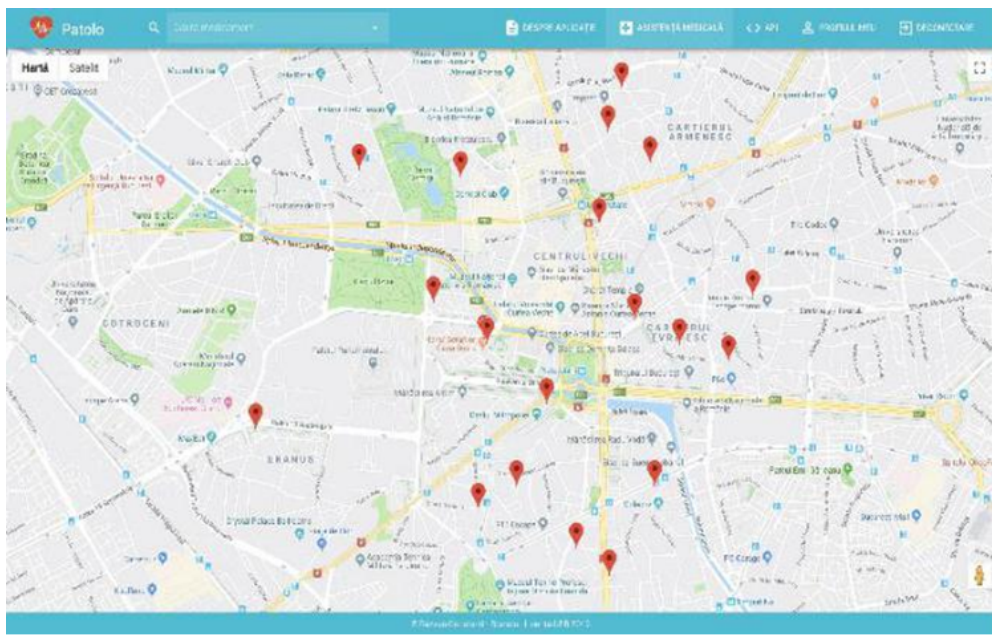

Fig. 10. Patolo-medical assistance finder

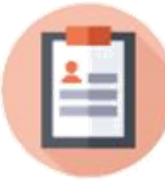

Cetall cont

Razvan Stanciu

Înregistrat la data: 20.04 .2019

Medicamente salvate

Altargo

Lovit, crema

Biazol, crema

Allergodil naseu spray, aerosol

Fig. 11. Patolo-user profile page

The last feature is written so that developers can use the medicines from the database in order to develop other med- ical applications. This will save them a lot of time and resources from having to build their own database.

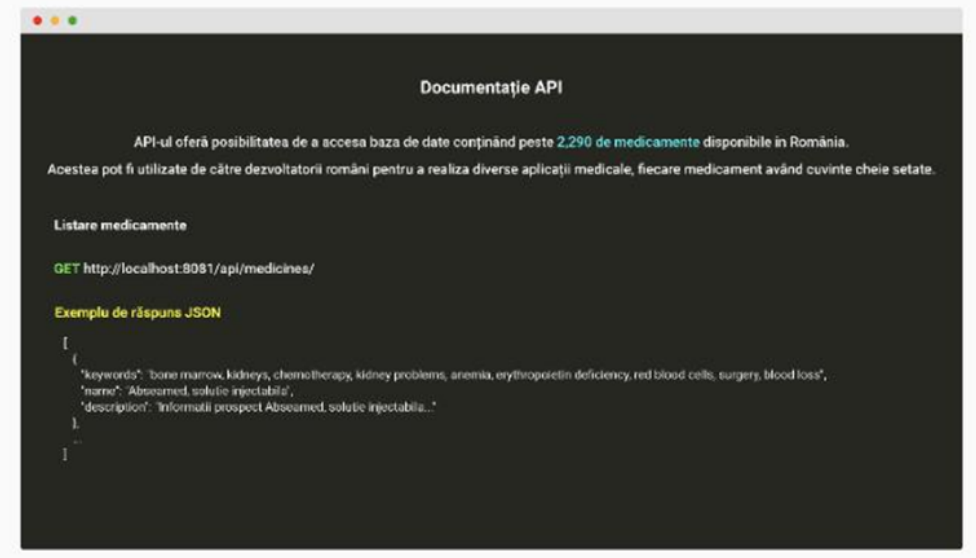

Fig. 12. Patolo-API documentation page 


\section{CONCLUSION AND FUTURE WORK}

Technological innovations drastically changed and improved our quality of life. There are many domains which benefit from the evolution of technology but one of the most important fields is healthcare.

Advances in healthcare are extremely important as there are many issues that require our attention. The field of medicine is a sensitive topic due to the fact that one mistake could lead to the loss of countless human lives. It was a long and difficult journey to reach the current state of medicine, but one thing is quite clear, technology was and will remain essential to the evolution of the healthcare system.

Patolo is a medical tool that does not require any prior medical knowledge and that is useful for self-diagnosis and treatment recommendation. Using the application is extremely easy and fast, because no healthcare surveys or personal information are necessary in order to complete the diagnosis procedure, so it can save a lot of time for the user. In ad- dition to the main use case, by building the database for medicines, the application now provides developers with a public API that can be used to develop other healthcarerelated products. Due to the fact that Patolo is based on keywords, the volume of data is essential to increase the accuracy.

At the moment, the accuracy is at a decent level but there are a few things that can be done in order to increase it. Firstly, custom translation could solve the issue of problematic words. A problematic word is a word which loses its meaning when is translated from one language to another. Secondly, the number of diseases in the database should also be increased.

To sum up, the solution proposed in this paper can still be improved and that is why feedback is vital. In the near future, Patolo might get in touch with healthcare professionals for ideas to bring new useful features and services to the users.

\section{REFERENCES}

[1] H. Sampathkumar, X.-W. Chen, and B. Luo, "Ontology-based visualization of healthcare data mined from online healthcare forums," in International Conference on Healthcare Informatics, Dallas, TX, 2015. doi: https://doi.org/10.1109/ ichi.2015.46

[2] A. Al-Canaan and A. Khoumsi, "Towards designing high-performance restful multimedia web services on FPGA," Journal of Advances in Technology and Engineering Studies, vol. 4, no. 3, pp. 111-117, 2018. doi: https://doi.org/10.20474/ jater-4.3.2

[3] C. Pasupathi and V. Kalavakonda, "Evidence based health care system using big data for disease diagnosis," in 2nd International Conference on Advances in Electrical, Electronics, Information, Communication and Bio-Informatics, Chennai, India, 2016. doi: https://doi.org/10.1109/aeeicb.2016.7538393

[4] S. D. S. F. Marino, E. and C. Vadala, “A web serverless architecture for buildings modeling," International Journal of Technology and Engineering Studies, vol. 3, no. 3, pp. 93-100, 2017. doi: https://doi.org/10.20469/ijtes.3.40001-3

[5] K. Osei-Frimpong, A. Wilson, and F. Lemke, "Patient co-creation activities in healthcare service delivery at the micro level: The influence of online access to healthcare information," Technological Forecasting and Social Change, vol. 126, pp. 14-27, 2018. doi: https://doi.org/10.1016/j.techfore.2016.04.009

[6] P. Groves, B. Kayyali, D. Knott, and S. V. Kuiken, 'The'big data'revolution in healthcare: Accelerating value and innovation," Center for US Health System Reform Business Technology Office, New York, NY, Tech. Rep., 2016.

[7] J. Chen, K. Li, H. Rong, K. Bilal, N. Yang, and K. Li, "A disease diagnosis and treatment recommendation system based on big data mining and cloud computing," Information Sciences, vol. 435, pp. 124-149, 2018. doi: https://doi.org/10. 1016/j.ins.2018.01.001

[8] P.-J. Kwon, H. Kim, and U. Kim, "A study on the web-based intelligent self-diagnosis medical system," Advances in Engineering Software, vol. 40, no. 6, pp. 402-406, 2009. doi: https://doi.org/10.1016/j.advengsoft.2008.07.004

[9] M. A. Hasan, A. R. Chowdhury et al., "Human disease diagnosis using a fuzzy expert system," Journal of Computing, vol. 2, no. 6, pp. 66-70, 2010.

[10] A. Kampouraki, D. Vassis, P. Belsis, and C. Skourlas, "E-Doctor: A web based support vector machine for automatic medical diagnosis," Procedia-Social and Behavioral Sciences, vol. 73, pp. 467-474, 2013. doi: https://doi.org/10.1016/ j.sbspro.2013.02.078

[11] B. Savkovic, P. Kovac, I. Mankova, M. Gostimirovic, K. Rokosz, and D. Rodic, "Surface roughness modeling of semi solid aluminum milling by fuzzy logic," Journal of Advances in Technology and Engineering Studies, vol. 3, no. 2, pp. 51-63, 2017. doi: https://doi.org/10.20474/jater-3.2.2 
[12] A. N. Noorzad. and T. Sato, "Multi-criteria fuzzy-based handover decision system for heterogeneous wireless networks," International Journal of Technology and Engineering Studies, vol. 3, no. 4, pp. 159-168, 2017. doi: https://doi.org/10. 20469/ijtes.3.40004-4

[13] F. Ricci, L. Rokach, and B. Shapira, "Recommender systems: Introduction and challenges," in Recommender Systems Handbook. Boston, MA: Springer, 2015.

[14] G. Adomavicius and A. Tuzhilin, "Toward the next generation of recommender systems: A survey of the state-of-the-art and possible extensions," IEEE Transactions on Knowledge \& Data Engineering, no. 6, pp. 734-749, 2005. doi: https: //doi.org/10.1109/tkde.2005.99

[15] M. Wiesner and D. Pfeifer, "Health recommender systems: Concepts, requirements, technical basics and challenges," International Journal of Environmental Research and Public Health, vol. 11, no. 3, pp. 2580-2607, 2014. doi: https: //doi.org/10.3390/ijerph110302580

[16] Y. Zhang, D. Zhang, M. M. Hassan, A. Alamri, and L. Peng, "CADRE: Cloud-assisted drug recommendation service for online pharmacies," Mobile Networks and Applications, vol. 20, no. 3, pp. 348-355, 2015. doi: https://doi.org/10.1007/ s11036-014-0537-4

[17] K. Shimada, H. Takada, S. Mitsuyama, H. Ban, H. Matsuo, H. Otake, H. Kunishima, K. Kanemitsu, and M. Kaku, "Drugrecommendation system for patients with infectious diseases," in AMIA Annual Symposium Proceedings, New York, NY, 2005.

[18] J. Soni, U. Ansari, D. Sharma, and S. Soni, "Predictive data mining for medical diagnosis: An overview of heart disease prediction," International Journal of Computer Applications, vol. 17, no. 8, pp. 43-48, 2011. doi: https://doi.org/10. 5120/2237-2860

[19] R. M. Galimova, I. V. Buzaev, K. A. Ramilevich, L. K. Yuldybaev, and A. F. Shaykhulova, “Artificial intelligence: Developments in medicine in the last two years," Chronic Diseases and Translational Medicine, vol. 5, no. 1, pp. 64-70, 2019. doi: https://doi.org/10.1016/j.cdtm.2018.11.004

[20] P. Hamet and J. Tremblay, "Artificial intelligence in medicine," Metabolism, vol. 69, pp. S36-S40, 2017. doi: https://doi. org/10.1016/j.metabol.2017.01.011

[21] Z. Obermeyer and E. J. Emanuel, "Predicting the future big data, machine learning, and clinical medicine," The New England Journal of Medicine, vol. 375, no. 13, pp. 12-16, 2016. doi: https://doi.org/10.1056/nejmp1606181

[22] V. H. Buch, I. Ahmed, and M. Maruthappu, "Artificial intelligence in medicine: Current trends and future possibilities," British Journal of General Practice, vol. 68, no. 668, pp. 143-144, 2018. doi: https://doi.org/10.3399/bjgp18x695213

[23] J. H. Chen and S. M. Asch, "Machine learning and prediction in medicine beyond the peak of inflated expectations," The New England Journal of Medicine, vol. 376, no. 26, pp. 25-37, 2017. doi: https://doi.org/10.1056/nejmp1702071

[24] R. Miotto, F. Wang, S. Wang, X. Jiang, and J. T. Dudley, "Deep learning for healthcare: Review, opportunities and challenges," Briefings in Bioinformatics, vol. 19, no. 6, pp. 1236-1246, 2017. doi: https://doi.org/10.1093/bib/bbx044

[25] E. Mehraeen, M. Ghazisaeedi, J. Farzi, and S. Mirshekari, "Security challenges in healthcare cloud computing: A systematic," Global Journal of Health Science, vol. 9, no. 3, pp. 157-168, 2017. doi: https://doi.org/10.5539/gjhs.v9n3p157

[26] S. Ashtari, A. Eydgahi, and H. Lee, "Exploring cloud computing implementation issues in healthcare industry," in Transactions of the International Conference on Health Information Technology Advancement, New York, NY, 2015.

[27] Y. Jalghoum and S. Khasawneh, "An empirical research of the challenges to E-health initiative in Jordan," International Journal of Business and Social Sciences, vol. 7, no. 11, pp. 51-65, 2016.

[28] Y. Yang, X. Zhang, and P. K. Lee, "Improving the effectiveness of online healthcare platforms: An empirical study with multi-period patient-doctor consultation data," International Journal of Production Economics, vol. 207, pp. 70-80, 2019. doi: https://doi.org/10.1016/j.ijpe.2018.11.009 